\title{
Retrospective analysis of mortality and Candida isolates of 75 patients with candidemia: a single hospital experience
}

This article was published in the following Dove Press journal:

Infection and Drug Resistance

8 July 2015

Number of times this article has been viewed

\author{
Ryuichi Hirano' \\ Yuichi Sakamoto ${ }^{2}$ \\ Kumiko Kudo' \\ Motoki Ohnishi ${ }^{3}$ \\ 'Department of Pharmacy, Aomori \\ Prefectural Central Hospital, \\ Aomori, Japan; ${ }^{2}$ Laboratory Medicine \\ and Blood transfusion, Aomori \\ Prefectural Central Hospital, Aomori, \\ Japan; ${ }^{3}$ General Medicine, Aomori \\ Prefectural Central Hospital, Aomori, \\ Japan
}

\begin{abstract}
The mortality rate for candidemia is approximately $30 \%-60 \%$. However, prognostic factors in patients with candidemia have not yet been elucidated in detail. The aim of the present study was to analyze prognostic factors for candidemia using the mortality rate and Candida isolates of patients with candidemia. Seventy-five patients with candidemia were analyzed between January 2007 and December 2013. The main outcome of this study was the 30-day mortality rate after the diagnosis of candidemia. The acute physiology and chronic health evaluation II score (APACHE II score) was measured in 34 patients (45.3\%). Odds ratios (ORs) for death due to candidemia were analyzed using a multivariate stepwise logistic regression analysis. Twenty (26.6\%) patients died within 30 days of being diagnosed with candidemia. Non-survivors had a significantly higher APACHE II score $(n=7$, mean; 18.9 4 4.5) than that of survivors $(n=27$, mean; 14.0 \pm 5.0$)$. Advanced age $(\mathrm{OR}=1.1,95 \%$ confidence interval $=1.01-1.23, P=0.04)$ was a significant risk factor for a high mortality rate, whereas removal of a central venous catheter $(\mathrm{OR}=0.03,95 \%$ confidence interval $=0.002-0.3, P=0.01)$ was associated with a lower mortality rate. Seventy-six Candida spp. were isolated from blood cultures: Candida albicans 28 (36.8\%), Candida parapsilosis 23 (30.2\%), Candida guilliermondii 16 (21.0\%), Candida glabrata four (5.2\%), Candida tropicalis two (2.6\%), and Candida spp. three (3.9\%) that could not be identified. C. parapsilosis was the most frequently isolated species in younger patients ( $<65$ years), whereas C. albicans was the most frequently isolated in elderly patients ( $\geq 65$ years). Physicians who treat candidemia need to consider removing the central venous catheter and pay attention to the general condition of patients, particularly that of elderly patients.
\end{abstract}

Keywords: aging, Candida, candidemia, central venous catheter, Japan

\section{Introduction}

Candida spp. are ranked as the fourth main cause of bloodstream infections in hospitals, and the frequency of candidemia has increased in recent years. ${ }^{1}$ The mortality rate for candidemia is approximately $30 \%-60 \%$, and the prognosis is generally poor. ${ }^{2}$ Invasive medical technologies, such as the use of immunosuppressive agents or anticancer drugs via central venous catheters (CVCs), are associated with the development of candidemia. ${ }^{3}$ Therefore, an early diagnosis and treatment are more important for improving the outcome of patients with candidemia due to the increased use of invasive medical technologies in recent years. ${ }^{4}$

Fifteen Candida spp. have been isolated from inpatients ${ }^{3}$ and the susceptibilities of these species to various antifungal agents differ, with Candida albicans being sensitive to many antifungal agents. Among the non-albicans Candida spp., Candida parapsilosis is resistant to micafungin, and Candida glabrata exhibits low sensitivity to many azole antifungals. The proportions of Candida isolates also vary among regions. C. glabrata
Correspondence: Ryuichi Hirano Department of Pharmacy, Aomori Prefectural Central Hospital, 2-I-I Higashi-tsukurimichi, Aomori-shi, Aomori 030 8553, Japan

Tel $+8 \mid 177268007$

Fax +8I 177268325

Email r.hiranolII5@gmail.com 
is generally more common in Western countries, whereas Candida tropicalis is more common in Asia. ${ }^{3}$ A surveillance study conducted between 2001 and 2002, a time during which echinocandin antifungal agents were not used in Japan, isolated C. albicans, C. parapsilosis, and C. glabrata from 40\%, 23\%, and $18 \%$ of candidemia cases, respectively. ${ }^{5}$ Forrest et al reported correlations between the increased use of echinocandin antifungal agents and higher incidence of $C$. parapsilosis candidemia, as well as with reductions in $C$. tropicalis and C. glabrata candidemia. ${ }^{6}$ However, difficulties are associated with basing strategies on the findings of previous surveillance studies because some reported that the emergence of echinocandin antifungal agents changed the proportions of different Candida spp. isolates in candidemia. ${ }^{6,7}$ If a yeast-like fungus is isolated from a blood culture, early empirical treatment must be initiated to improve the outcome of the patient; ${ }^{4}$ however, the antifungal agents recommended for the treatment of candidemia vary according to the species isolated. Since few studies have been conducted on the prognostic factors of candidemia mortality in Japan, they remain unclear in patients with candidemia. Thus, the treatment of candidemia needs to be optimized to investigate the risk factors for death and determine the proportions of different isolates in each hospital.

In the present study, we performed a retrospective analysis of the candidemia cases that occurred in our hospital in order to identify the prognostic factors of and proportions of different isolates in candidemia.

\section{Materials and methods Study design and population}

Seventy-five patients diagnosed with candidemia in Aomori Prefectural Central Hospital between January 2007 and December 2013 were enrolled in this study. This is a general hospital with 695 beds and is located in Aomori City, Japan. Based on medical records, we included patients with symptoms of infection (eg, fever up to $37^{\circ} \mathrm{C}$ ) and with at least one positive blood culture for Candida spp. Patients without symptoms of infection were excluded from this study. The main outcome of the present study was the 30-day mortality rate after the diagnosis of candidemia, as described previously. ${ }^{8-10}$ The study protocol was approved by the Ethics Committee of Aomori Prefectural Central Hospital. Investigations were carried out by securing each patient's anonymity.

\section{Definitions of each disease} and clinical data collection

We assessed age, sex, complications, main disease, and antibiotics used. We confirmed in patients with CVC whether catheters were removed after the diagnosis of candidemia. We calculated the acute physiology and chronic health evaluation II score (APACHE II score) of 34 (45.3\%) patients. $^{11}$ Candida endophthalmitis was diagnosed by an ophthalmologist based on the finding of fungal endophthalmitis.

We defined each disease state as follows. Patients who received cyclosporine, tacrolimus, anticancer drugs, or corticosteroids were defined as immunosuppression. Patients who had a neutrophil count $<500$ cell $/ \mu \mathrm{L}$ at the time of the blood culture were defined as neutropenia. Patients who required dialysis or those with serum creatinine values $\geq 3.0 \mathrm{mg} / \mathrm{dL}$ were defined as renal failure. Patients who had more than five times the upper limit for aspartate transaminase, alanine transaminase, or gamma glutamyl transpeptidase were defined as liver failure. Patients who had underlying diseases such as asthma, pneumonia, lung abscess, and lung cancer were defined as lung disease. Patients who were 65 years of age or older were defined as elderly patients, whereas patients who were 64 years of age or younger were defined as younger patients. The cut-off value of age was determined by the median age of all patients.

\section{Mycological data collection}

Mycological data were collected from the records of each patient's blood culture tests. Blood culture tests were performed using a series of aerobic and anaerobic blood culture bottles with Bac T/ALERT, which were incubated in a Bac T/ALERT 3D blood culture system (SYSMEX; bioMerieux, Lyon, France). After Gram staining, blood cultures that tested positive for yeasts were subcultured on CHROM agar Candida culture medium (Kanto Chemical, Tokyo, Japan) and incubated for 48 hours at $30^{\circ} \mathrm{C}$. Candida spp. were identified using the ID 32C system (SYSMEX; bioMerieux), and antifungal susceptibility tests were performed using the yeast-like fungal drug sensitivity kit ASTY (Kyokuto Pharmaceutical industrial, Tokyo, Japan). Resistance to fluconazole and micafungin were determined based on the minimum inhibitory concentration (MIC) after incubating for 48 hours at $35^{\circ} \mathrm{C}$. We used species-specific antifungal drug susceptibility break points for fluconazole and micafungin according to the Clinical and Laboratory Standards Institute (CLSI) M27-S4. ${ }^{12}$ Since there is currently no break point for fluconazole in Candida guilliermondii, epidemiological cut-off values (ECVs) were applied to determine susceptibility. ${ }^{13}$ We defined three Candida spp. that could not be identified as others. ECVs for C. guilliermondii were also applied to others to determine susceptibility. ${ }^{14}$ The ECVs for $C$. guilliermondii were as follows; resistant strains were defined as MIC $\geq 16 \mu \mathrm{g} / \mathrm{mL}$ for fluconazole and $\mathrm{MIC} \geq 4 \mu \mathrm{g} / \mathrm{mL}$ for micafungin. ${ }^{14}$ 


\section{Statistical analysis}

Results were expressed as mean values \pm standard deviation. Continuous data were analyzed using the Mann-Whitney $U$ test, and categorical data were analyzed using the $\chi^{2}$ test. $P<0.05$ was considered significant. We divided patients into survivors and non-survivors based on death within 30 days of being diagnosed with candidemia, and we evaluated the factors associated with death by comparing the treatment and clinical factors. Significant variants in the univariate analysis were selected for inclusion in a multivariate stepwise logistic regression analysis. We included age, the removal of CVC, lung disease, and infection with C. albicans and C.parapsilosis in a multivariate stepwise logistic regression analysis in order to determine the odds ratios (ORs) for death within 30 days of being diagnosed with candidemia. The factors associated with death among elderly patients by comparing the treatment and clinical factors between younger and elderly patients were also evaluated. We included immunosuppression therapy in a multivariate stepwise logistic regression analysis to determine the ORs for death among elderly patients with candidemia. We did not include the APACHE II score in a multivariate stepwise logistic regression analysis because it could not be calculated in more than half of all patients. All statistical analyses were performed using the Excel- Toukei 2012 (Social Survey Research Information Co, Ltd, Tokyo, Japan).

\section{Results}

\section{Patient characteristics}

Seventy-five patients were included in this study (51 males and 24 females, mean age; 62.1 \pm 19.4 , age range; 5-94, age median; 65). Patient demographics, underlying diseases, diagnoses, and treatments for candidemia are shown in Table 1 . Twenty (26.6\%) patients died within 30 days of being diagnosed with candidemia. We calculated the APACHE II score of 34 (45.3\%) patients (mean; 15.0 5 5.2, range; $6-27$, median; 16.5 ). A total of $92 \%$ of patients had undergone central venous catheterization, $77.3 \%$ of patients required total parenteral nutrition therapy, and $90.6 \%$ of patients had low serum albumin levels.

\section{Diagnosis and treatment of candidemia}

In order to diagnose candidemia, two sets of blood cultures were obtained from $72 \%$ of patients. The serum $\beta$-D glucan level was examined in $70.6 \%$ of cases. A diagnosis of endophthalmitis due to Candida spp. was obtained in $44 \%$ of patients. Among the patients who received an ophthalmological consultation, two were diagnosed with Candida endophthalmitis caused by C. albicans. The CVC removal
Table I Characteristics of patients with candidemia

\begin{tabular}{|c|c|c|}
\hline Characteristics & $\mathbf{N}$ & $\%$ \\
\hline Number of cases & 75 & \\
\hline Age (range) & $62.1(5-94)$ & \\
\hline Sex (male) & 51 & 68 \\
\hline Death within 30 days of candidemia diagnosis & 20 & 26.6 \\
\hline Presence of CVC & 69 & 92 \\
\hline Immunosuppressive therapy & 30 & 40 \\
\hline Intensive care unit admission & 7 & 9.3 \\
\hline Total parenteral nutrition & 58 & 77.3 \\
\hline Mechanical ventilation & 7 & 9.3 \\
\hline Renal failure & 6 & 8 \\
\hline Liver failure & 20 & 26.6 \\
\hline Low serum albumin (<2.5 mg/dL) & 68 & 90.6 \\
\hline Organ transplantation & 7 & 9.3 \\
\hline History of Candida colonization & 7 & 9.3 \\
\hline Surgery & 20 & 26.6 \\
\hline Neutropenia & 6 & 8 \\
\hline Malignancy & 41 & 54.6 \\
\hline Lung disease & 14 & 18.6 \\
\hline Diabetes mellitus & 16 & 21.3 \\
\hline \multicolumn{3}{|l|}{ Diagnosis of and treatment for candidemia } \\
\hline Two sets of blood cultures & 54 & 72 \\
\hline Examination of serum $\beta$-D glucan & 53 & 70.6 \\
\hline Diagnosis of endophthalmitis due to Candida spp. & 33 & 44 \\
\hline Removal of CVC & 49 & 65.3 \\
\hline \multicolumn{3}{|l|}{ Antifungal agents } \\
\hline Fosfluconazole & 28 & 37.3 \\
\hline Micafungin & 34 & 45.3 \\
\hline Voriconazole & 2 & 2.6 \\
\hline Liposomal amphotericin B & 7 & 9.3 \\
\hline Itraconazole & 2 & 2.6 \\
\hline
\end{tabular}

Abbreviation: CVC, central venous catheter.

rate was $65.3 \%$. Among the 73 patients who received antifungal agents, the treatments employed micafungin, fosfluconazole, liposomal amphotericin B, voriconazole, and itraconazole in 34 (45.3\%), 28 (37.3\%), seven (9.3\%), two (2.6\%), and two (2.6\%) cases, respectively.

\section{Mycological data of Candida spp.}

Among the 76 Candida isolates from blood cultures, C. albicans accounted for 28 (36.8\%), C. parapsilosis 23 (30.2\%), C. guilliermondii 16 (21\%), C. glabrata four $(5.2 \%)$, C. tropicalis two $(2.6 \%)$, and others three $(3.9 \%)$. The number of total Candida isolates was 76 because one patient was diagnosed with a mixed infection of $C$. parapsilosis and C. guilliermondii. Of the 76 Candida isolates, eight strains (10.5\%) exhibited resistance to fluconazole, ie, C. parapsilosis, two (8.6\%); C. guilliermondii, two (12.5\%); C. tropicalis, two (100\%); and others, two (66.6\%). Five strains (6.6\%) were resistant to micafungin, ie, C. albicans (3.5\%); C. parapsilosis (4.3\%); C. guilliermondii (6.2\%); C. glabrata (25\%); and others (33.3\%). 


\section{Factors associated with death}

Patients were divided into survivors and non-survivors according to their 30-day mortality rates (Table 2). Nonsurvivors were older and had a higher rate of infection with C. albicans and lung disease than survivors. Non-survivors had a lower rate of CVC removal and lower rate of infection with $C$. parapsilosis than survivors. Non-survivors had a significantly higher APACHE II score ( $n=7$, mean; 18.9 \pm 4.5$)$ than that of survivors $(n=27$, mean; 14.0 \pm 5.0$)$ according to the Mann-Whitney $U$ test $(P=0.02)$. The multivariate analysis identified two factors that had significant relationships with death due to candidemia: advanced age $(\mathrm{OR}=1.1,95 \%$ confidence interval $[\mathrm{CI}]=1.01-1.23, P=0.04)$ was associated with a higher mortality rate than the removal of $\mathrm{CVC}(\mathrm{OR}=0.03$, 95\% CI $=0.002-0.3, P=0.01$ ) (Table 3 ). We divided patients into two groups to investigate the risk factors for death among

Table 2 Comparison of various parameters among survivors and non-survivors

\begin{tabular}{|c|c|c|c|}
\hline & $\begin{array}{l}\text { Survivors } \\
\text { (\%) }\end{array}$ & $\begin{array}{l}\text { Non- } \\
\text { survivors (\%) }\end{array}$ & $P$-value \\
\hline \multicolumn{4}{|l|}{ Clinical demographics } \\
\hline Number of cases & 55 & 20 & \\
\hline Age* & $59.1 \pm 20.6$ & $70.5 \pm 11.5$ & 0.03 \\
\hline Sex (male) & 39 & 12 & 0.37 \\
\hline Presence of CVC & $52(94.5)$ & $17(85)$ & 0.33 \\
\hline Immunosuppressive therapy & $21(38.1)$ & $9(45)$ & 0.59 \\
\hline Intensive care unit admission & $5(9.1)$ & $2(10)$ & 0.9 \\
\hline Total parenteral nutrition & $43(78.1)$ & $15(75)$ & 0.76 \\
\hline Mechanical ventilation & $4(7.3)$ & $3(15)$ & 0.37 \\
\hline Renal failure & $4(7.3)$ & $2(10)$ & 0.7 \\
\hline Liver failure & $12(21.8)$ & $8(40)$ & 0.11 \\
\hline Low albumin $(<2.5 \mathrm{mg} / \mathrm{dL})$ & $49(89.0)$ & $19(95)$ & 0.43 \\
\hline Organ transplantation & $4(7.3)$ & $3(15)$ & 0.3 \\
\hline History of Candida colonization & $4(7.3)$ & $3(15)$ & 0.37 \\
\hline Surgery & $16(29.1)$ & $4(20)$ & 0.56 \\
\hline Neutropenia & $3(5.5)$ & $3(15)$ & 0.18 \\
\hline Malignancy & $32(58.1)$ & $9(45)$ & 0.31 \\
\hline Lung disease* & $7(12.7)$ & $7(35)$ & 0.028 \\
\hline Diabetes mellitus & II (20) & $5(25)$ & 0.64 \\
\hline \multicolumn{4}{|l|}{ Treatment for candidemia } \\
\hline Removal of CVC* & $44(80)$ & $5(25)$ & $<0.01$ \\
\hline Fosfluconazole & $21(38.1)$ & $7(35)$ & 0.8 \\
\hline Micafungin & $27(49.0)$ & $7(35)$ & 0.27 \\
\hline Liposomal amphotericin B & $5(9.0)$ & $2(10)$ & 0.9 \\
\hline \multicolumn{4}{|l|}{ Candida spp. } \\
\hline Candida albicans* & $16(29.0)$ & $12(60)$ & 0.023 \\
\hline Candida parapsilosis* & $22(40)$ & I (5) & $<0.01$ \\
\hline Candida guilliermondii & $13(23.6)$ & $3(15)$ & 0.51 \\
\hline Resistance to fluconazole & $4(7.2)$ & $4(20)$ & 0.11 \\
\hline Resistance to micafungin & $4(7.2)$ & I (5) & 0.72 \\
\hline
\end{tabular}

Notes: Data are expressed as the mean \pm standard deviation (SD; min-max). Statistical analyses were performed using the $\chi^{2}$ test or Mann-Whitney $U$ test. *Significant difference $(P<0.05)$.

Abbreviation: CVC, central venous catheter.
Table 3 Risk factors for death within 30 days of being diagnosed with candidemia according to a stepwise regression analysis

\begin{tabular}{llll}
\hline Risk factors & Odds ratio & $\mathbf{9 5 \%} \mathbf{~ C l}$ & $P$-value \\
\hline Age & 1.11 & $1.01-1.23$ & 0.04 \\
Removal of CVC & 0.03 & $0.002-0.3$ & 0.01 \\
Lung disease & 3.99 & $0.6-26.4$ & 0.15 \\
Candida albicans & 6.2 & $0.4-96.7$ & 0.19 \\
Candida parapsilosis & 0.12 & $0.005-2.9$ & 0.19 \\
\hline
\end{tabular}

Notes: A multivariate analysis was performed using a stepwise logistic regression model. Each factor was selected by a univariate analysis between survivors and nonsurvivors $(P<0.05)$.

Abbreviations: $\mathrm{CVC}$, central venous catheter; $\mathrm{Cl}$, confidence interval.

elderly patients with candidemia because the multivariate logistic regression analysis identified advanced age as a significant risk factor for death. We compared parameters between younger and elderly patients (Table 4). The mortality rate of candidemia was higher in elderly patients $(33.3 \%$, $14 / 42)$ than in younger patients $(18.1 \%, 6 / 33)$; however, the $\chi^{2}$ analysis showed that this difference was not significant $(P=0.14)$. The rates of immunosuppression therapy and organ transplantation were lower in elderly patients than in younger patients. The Mann-Whitney $U$ test revealed that elderly patients had a significantly higher APACHE II score $(\mathrm{n}=21$, mean; $16.4 \pm 4.9)$ than that of younger patients $(\mathrm{n}=13$, mean; 12.7 \pm 5.0$)(P=0.04)$. No significant factors for death among elderly patients were identified in the multivariate analysis: immunosuppression therapy ( $\mathrm{OR}=2.5,95 \% \mathrm{CI}$ $=0.59-10.9, P=0.2$ ).

The 30-day mortality rates for each Candida spp. were as follows: C. albicans, $42.9 \%$ (12/28); C. parapsilosis, 4.3\% (1/23); C. guilliermondii, 18.7\% (3/16); C. glabrata, 25\% (1/4); C. tropicalis, $100 \%$ (2/2); others, $33.3 \%(1 / 3)$. The $\chi^{2}$ analysis revealed a significant difference in mortality rates between patients with $C$. albicans and $C$. parapsilosis $(P<0.01)$.

\section{Discussion}

Since candidemia is a nosocomial infection with a high mortality rate, ${ }^{8-10}$ it is important to optimize its treatment based on an accurate diagnosis and by determining the proportions of different isolates in each hospital. In the present study, the 30-day mortality rate of patients with candidemia was approximately $26 \%$, which was consistent with previous findings. ${ }^{8,9}$

Approximately $20 \%$ of patients with candidemia develop Candida endophthalmitis. ${ }^{15}$ In the present study, approximately $6 \%$ patients were diagnosed with Candida endophthalmitis, which is lower than that of previous findings. C. albicans has been identified as the species that 
Table 4 Comparison of various parameters among younger and elderly patients with candidemia

\begin{tabular}{|c|c|c|c|}
\hline & $\begin{array}{l}\text { Younger } \\
\text { patients (\%) }\end{array}$ & $\begin{array}{l}\text { Elderly } \\
\text { patients (\%) }\end{array}$ & $P$-value \\
\hline \multicolumn{4}{|l|}{ Clinical demographics } \\
\hline Number of cases & 33 & 42 & \\
\hline Age* & $44.1 \pm 14.4$ & $76.3 \pm 7.2$ & $<0.01$ \\
\hline Sex (male) & 22 & 29 & 0.82 \\
\hline \multicolumn{4}{|l|}{ of candidemia diagnosis } \\
\hline Presence of CVC & $31(93.9)$ & $38(90.4)$ & 0.58 \\
\hline Immunosuppressive therapy* & $20(60.6)$ & $10(23.8)$ & $<0.01$ \\
\hline Intensive care unit admission & $2(6.0)$ & $5(11.9)$ & 0.45 \\
\hline Total parenteral nutrition & $25(75.7)$ & $33(78.5)$ & 0.72 \\
\hline Mechanical ventilation & $2(6.0)$ & $5(11.9)$ & 0.45 \\
\hline Renal failure & $2(6.0)$ & $4(9.5)$ & 0.68 \\
\hline Liver failure & II (33.3) & $9(21.4)$ & 0.24 \\
\hline Low albumin $(<2.5 \mathrm{mg} / \mathrm{dL})$ & $28(84.8)$ & $40(95.2)$ & 0.12 \\
\hline Organ transplantation* & $7(2 \mid .2)$ & $0(0)$ & $<0.01$ \\
\hline $\begin{array}{l}\text { History of Candida } \\
\text { colonization }\end{array}$ & $5(15.1)$ & $2(4.7)$ & 0.12 \\
\hline Surgery & $8(24.2)$ & $12(28.5)$ & 0.67 \\
\hline Neutropenia & $5(15.1)$ & $\mathrm{I}(2.3)$ & 0.08 \\
\hline Malignancy & $18(54.5)$ & $23(54.7)$ & 0.98 \\
\hline Lung disease & $5(I 5 . I)$ & $9(21.4)$ & 0.48 \\
\hline Diabetes mellitus & $6(18.1)$ & $10(23.8)$ & 0.58 \\
\hline \multicolumn{4}{|l|}{ Treatment for candidemia } \\
\hline Removal of CVC & $23(69.6)$ & $26(61.9)$ & 0.48 \\
\hline Fosfluconazole & II (33.3) & $17(40.4)$ & 0.52 \\
\hline Micafungin & $15(45.4)$ & $19(45.2)$ & 0.98 \\
\hline Liposomal amphotericin B & $3(9.0)$ & $4(9.5)$ & I \\
\hline \multicolumn{4}{|l|}{ Candida species } \\
\hline Candida albicans & $10(30.3)$ & $18(42.8)$ & 0.26 \\
\hline Candida parapsilosis & $12(36.3)$ & II (26.I) & 0.34 \\
\hline Candida guilliermondii & $6(18.1)$ & $10(23.8)$ & 0.55 \\
\hline Resistance to fluconazole & $5(15.1)$ & $3(7.1)$ & 0.29 \\
\hline Resistance to micafungin & $3(9.1)$ & $2(4.7)$ & 0.64 \\
\hline
\end{tabular}

Notes: Data are expressed as the mean \pm standard deviation (SD; min-max). Statistical analyses were performed using the $\chi^{2}$ test or Mann-Whitney $U$ test. *Significant difference $(P<0.05)$.

Abbreviation: CVC, central venous catheter.

most frequently causes Candida endophthalmitis. ${ }^{15}$ If the diagnosis and treatment of endophthalmitis are delayed, the patient may become blind. According to the treatment guidelines of the Infectious Diseases Society of America, an ophthalmological consultation to diagnose endophthalmitis is strongly recommended for all patients with candidemia. ${ }^{16}$ The low rate of ophthalmological consultations was identified as a problem during the diagnosis of candidemia in our hospital.

Of the Candida spp. isolated from blood cultures in the present study, the frequency of $C$. parapsilosis was higher while that of $C$. glabrata was lower than previous findings reported in Japan. ${ }^{5}$ C. glabrata is generally sensitive to micafungin, whereas $C$. parapsilosis has a high MIC. ${ }^{5}$ Forrest et al reported that after the introduction of echinocandin antifungal agents, the frequency of $C$. parapsilosis isolates increased, whereas that of C. glabrata decreased. ${ }^{6}$ In our hospital, micafungin has been used since 2004, and this drug is administered as a therapeutic as well as preventive agent to patients with suspected fungal infections. Therefore, the widespread use of micafungin is considered to be responsible for the specific proportions of isolates detected in our hospital, which differ from previous findings. ${ }^{5}$ In the susceptibility tests using fluconazole and micafungin, eight strains were resistant to fluconazole (10.5\%), and five strains were resistant to micafungin (6.5\%), which is consistent with previous findings. ${ }^{17}$

We identified two factors that correlated with death due to candidemia: advanced age and a failure to remove CVC. Many cases of candidemia are attributable to CVC, and the prognosis of patients is improved by removing the focus of infection. ${ }^{8,18}$ Our results indicated that persistent CVC was detrimental to patients with candidemia.

Previous studies identified advanced age as a significant risk factor for death among patients with candidemia. ${ }^{8-10}$ However, few studies have investigated risk factors for death among elderly patients. We could not identify any significant risk factors for death among elderly patients with candidemia. Elderly patients with candidemia had markedly high APACHE II scores and were treated with mechanical ventilation, which were considered significant risk factors for death. ${ }^{19}$ In our study, non-survivors had significantly higher APACHE II scores than those of survivors, and a significant difference was observed in this score between elderly and younger patients. The APACHE II score is a severity of illness index that is affected by the general condition of patients. Considering these results, poor general condition was considered to be responsible for the high mortality rate observed among elderly patients with candidemia.

Previous studies suggested that the proportion of nonalbicans Candida spp. increases with age; C. glabrata was detected in $20 \%$ of patients aged $\geq 65$ years. ${ }^{20}$ Thus, our results differed from previous findings. Immunosuppression therapy has been identified as a risk factor for non-albicans candidemia. ${ }^{21}$ In the present study, the rate of immunosuppression therapy was lower in elderly patients. This clinical factor was different from previous findings. In addition to immunosuppression therapy, other factors (eg, a history of gastrointestinal surgery and antifungal exposure) have been also reported as risk factors for non-albicans candidemia. ${ }^{22}$ Neutropenia and immunosuppression therapy were previously identified as risk factors for death among patients 
with candidemia. ${ }^{8,23,24}$ However, these factors were not associated with a high mortality rate in our study. A meaningful statistics analysis could not be performed because of the insufficient number of patients, which was a limitation of this study.

The mortality rate of patients with a high APACHE II score was previously reported to be high..$^{8,10,24,25}$ In the present study, the APACHE II score was only obtained in 34 (45.3\%) patients due to the lack of clinical data. Our result was consistent with these studies since non-survivors had a significantly higher score than that of survivors. The limitations of this study were the multivariate logistic regression analysis for death without APACHE II scores and small number of cases.

In conclusion, we identified two factors that correlated with death due to candidemia: advanced age and a failure to remove CVC. Physicians who treat candidemia need to consider removing $\mathrm{CVC}$ and pay attention to the general condition of patients, particularly elderly patients. The results of the present study provide a clearer understanding of the epidemiology of candidemia in Japan.

\section{Acknowledgments}

We thank Naoki Tachibana (Chief doctor, Department of Laboratory Medicine and Blood transfusion, Aomori Prefectural Central Hospital) and Ryuichi Teshiromori (Medical Technologist, Department of Laboratory Medicine and Blood transfusion, Aomori Prefectural Central Hospital) for their help and comments on this study.

\section{Disclosure}

There are no conflicts of interest associated with this study.

\section{References}

1. Wisplinghoff H, Bischoff T, Tallent SM, Seifert H, Wenzel RP, Edmond MB. Nosocomial bloodstream infections in US hospitals: analysis of 24179 cases from a prospective nationwide surveillance study. Clin Infect Dis. 2004;39(3):309-317.

2. Flevari A, Theodorakopoulou M, Velegraki A, Armaganidis A, Dimopoulos G. Treatment of invasive candidiasis in the elderly: a review. Clin Interv Aging. 2013;8:1199-1208.

3. Yapar N. Epidemiology and risk factors for invasive candidiasis. Ther Clin Risk Manag. 2014;10:95-105.

4. Morrell M, Fraser VJ, Kollef MH. Delaying the empiric treatment of Candida bloodstream infection until positive blood culture results are obtained: a potential risk factor for hospital mortality. Antimicrob Agents Chemother. 2005;49(9):3640-3645.

5. Takakura S, Fujihara N, Saito T, Kudo T, Iinuma Y, Ichiyama S. National surveillance of species in Japan and their susceptibility to six antifungal agents including voriconazole and micafungin. JAntimicrob Chemother. 2004;53(2):283-289.

6. Forrest GN, Weekes E, Johnson JK. Increasing incidence of Candida parapsilosis candidemia with caspofunin usage. J Infect. 2008;56(2): 126-129.
7. Tarumoto N, Abe Y, Yamaguchi T, Maesaki S. Clinical aspects of candidemia before and after the introduction of micafungin in Saitama Medical School Hospital. Jpn J Chemother. 2010;58:14-17.

8. Andes DR, Safdar N, Baddley JW, et al. Impact of treatment strategy on outcomes in patients with candidemia and other forms of invasive candidiasis: a patient-level quantitative review of randomized trials. Clin Infect Dis. 2012;54(8):1110-1122.

9. Viscoli C, Girmenia C, Marinus A, et al. Candidemia in cancer patients: a prospective, multicenter surveillance study by the Invasive Fungal Infection Group (IFIG) of the European Organization for Research and Treatment of Cancer (EORTC). Clin Infect Dis. 1999;28(5):1071-1079.

10. Colombo AL, Guimaraes T, Sukienik T, et al. Prognostic factors and historical trends in the epidemiology of candidemia in critically ill patients: an analysis of five multicenter studies sequentially conducted over a 9-year period. Intensive Care Med. 2014;40(10):1489-1498.

11. Chang RW, Jacobs S, Lee B. Predicting outcome among intensive care unit patients using computerized trend analysis of daily Apache II scores corrected for organ system failure. Intensive Care Med. 1988;14(5): $558-566$.

12. Clinical and Laboratory Standards Institute. Reference method for dilution antifungal susceptibility testing of yeasts; 4th informational supplement. CLSI document M27-S4. Wayne, PA: Clinical and Laboratory Standards Institute; 2012.

13. Chen CY, Huang SY, Tang JL, et al. Clinical features of patients with infections caused by Candida guilliermondii and Candida fermentati and antifungal susceptibility of the isolates at a medical centre in Taiwan, 2001-2010. J Antimicrob Chemother. 2013;68(11):2632-2635.

14. Pfaller MA, Diekema DJ. Progress in antifungal susceptibility testing of Candida spp. By use of Clinical and Laboratory Standards Institute broth microdilution methods, 2010 to 2012. J Clin Microbiol. 2012;50(9):2846-2856.

15. Nagao M, Saito T, Doi S, et al. Clinical characteristics and risk factors of ocular candidiasis. Diagn Microbial Infect Dis. 2012;73(2):149-152.

16. Pappas PG, Kauffman CA, Andes D, et al. Clinical practice guidelines for the management of candidiasis: 2009 update by the Infectious Diseases Society of America. Clin Infect Dis. 2009;48(5):503-535.

17. Takakura S, Fujihara N, Saito T, et al. Clinical factors associated with fluconazole resistance and short-term survival in patients with Candida bloodstream infection. Eur J Cliln Microbiol Infect Dis. 2004;23(5):380-388.

18. Kollef M, Micek S, Hampton N, Doherty JA, Kumar A. Septic shock attributed to Candida infection: importance of empiric therapy and source control. Clin Infect Dis. 2012;54(12):1739-1746.

19. Wang H, Liu N, Yin M, et al. The epidemiology, antifungal use and risk factors of death in elderly patients with candidemia: a multicenter retrospective study. BMC Infect Dis. 2014;14:609.

20. Pfaller MA, Diekema DJ, Jones RN, Messer SA, Hollis RJ; SENTRY Participants Group. Trends in antifungal susceptibility of Candida spp. Isolated from pediatric and adult patients with bloodstream infections: SENTRY Antimicrobial Surveillance Program, 1997 to 2000. J Clin Microbiol. 2002;40(3):852-856.

21. Al-Rawahi GN, Roscoe DL. Ten-year review of candidemia in a Canadian tertiary care centre: Predominance of non-albicans Candida species. Can J Infect Dis Med Microbiol. 2013;24(3):e65-e68.

22. Playford EG, Marriott D, Nguyen Q, et al. Candidemia in nonneutropenic ill patients: risk factors for non-albicans Candida spp. Crit Care Med. 2008;36(7):2034-2039.

23. Anaissie EJ, Rex JH, Uzun O, Vartivarian S. Predictors of adverse outcome in cancer patients with candidemia. Am J Med. 1998;104(3): 238-245.

24. Viudes A, Pemán J, Cantón E, Ubeda P, López-Ribot JL, Gobernado M. Candidemia at a tertiary-care hospital: epidemiology, treatment, clinical outcome and risk factors for death. Eur J Clin Microbiol Infect Dis. 2002;21(11):767-774.

25. Chen YR, Lin LC, Young TG, Liu CE, Chen CH, Tsay RW. Risk factors for candidemia-related mortality at a medical center in central Taiwan. J Microbiol Immunol Infect. 2006;39(2):155-161. 
Infection and Drug Resistance

\section{Publish your work in this journal}

Infection and Drug Resistance is an international, peer-reviewed openaccess journal that focuses on the optimal treatment of infection (bacterial, fungal and viral) and the development and institution of preventive strategies to minimize the development and spread of resistance. The journal is specifically concerned with the epidemiology of antibiotic
Dovepress

resistance and the mechanisms of resistance development and diffusion in both hospitals and the community. The manuscript management system is completely online and includes a very quick and fair peerreview system, which is all easy to use. Visit http://www.dovepress.com/ testimonials.php to read real quotes from published authors.

Submit your manuscript here: http://www.dovepress.com/infection-and-drug-resistance-journal 